Utah State University

DigitalCommons@USU

$1-1-2012$

\title{
Stray Light Control for Asteroid Detection at Low Solar Elongation for the NEOSSat Micro-Satellite Telescope
}

Victor Isbrucker

John Stauder

Denis Laurin

Allan Hollinger

Follow this and additional works at: https://digitalcommons.usu.edu/sdl_pubs

\section{Recommended Citation}

Isbrucker, Victor; Stauder, John; Laurin, Denis; and Hollinger, Allan, "Stray Light Control for Asteroid Detection at Low Solar Elongation for the NEOSSat Micro-Satellite Telescope" (2012). Space Dynamics Lab Publications. Paper 61.

https://digitalcommons.usu.edu/sdl_pubs/61

This Article is brought to you for free and open access by the Space Dynamics Lab at DigitalCommons@USU. It has been accepted for inclusion in Space Dynamics Lab Publications by an authorized administrator of DigitalCommons@USU. For more information, please contact digitalcommons@usu.edu.

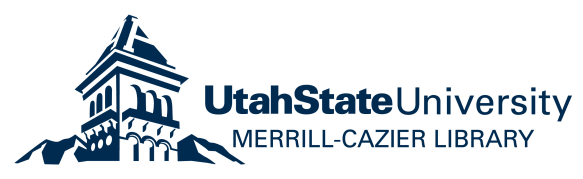




\title{
Stray light control for asteroid detection at low solar elongation for the NEOSSat micro-satellite telescope
}

\author{
Victor Isbrucker ${ }^{\mathrm{a}}$, John Stauder ${ }^{\mathrm{b}}$, Denis Laurin ${ }^{\mathrm{c}}$, Allan Hollinger ${ }^{\mathrm{c}}$ \\ ${ }^{\mathrm{a}}$ Isbrucker Consulting Inc., 4 Third Street, Sturgeon Point, Ontario, Canada, K0M 1N0; ${ }^{\mathrm{b}}$ Space \\ Dynamics Laboratory, Utah State University Research Foundation, 1695 North Research Park Way, \\ North Logan, Utah 84341, USA; ${ }^{\circ}$ Canadian Space Agency, 6767 route de l’Aéroport, St-Hubert, \\ Québec, Canada, J3Y 8Y9
}

\begin{abstract}
The Near Earth Object Surveillance Satellite (NEOSSat) is a small satellite dedicated to finding near Earth asteroids. Its surveying strategy consists of imaging areas of the sky to low solar elongation, while in a sun synchronous polar orbit (dawn-dusk). A high performance baffle will control stray light mainly due to Earth shine. Observation scenarios require solar shielding down to 45 degree solar elongation over a wide range of ecliptic latitudes. In order to detect the faintest objects (approx $20^{\text {th }} \mathrm{v}$ mag) given a $15 \mathrm{~cm}$ telescope and CCD detection system, background from stray light is a critical operational concern. The required attenuation is in the order of $10^{-12}$. The requirement was verified by analyses; testing was not attempted because the level of attenuation is difficult to measure reliably. We report consistent results of stray light optical modelling from two independent analyses. Launch is expected for late 2012.
\end{abstract}

Keywords: stray light analysis, baffle, space telescope, Earthshine, NEOSSat

\section{INTRODUCTION}

\subsection{The NEOSSat mission}

NEOSSat is a Canadian space telescope being developed in partnership between the Canadian Space Agency (CSA) and Defence Research and Development Canada (DRDC). Using a micro-satellite platform, NEOSSat has two operational objectives: 1) discover near-Earth asteroids (NEA) and 2) demonstrate the ability to produce tracking data on artificial satellites in high earth orbit. It is based on the highly successful Canadian MOST astronomy microsatellite (still operating since 2003) [1].

The CSA Mission Operations Centre will conduct the daily satellite operations. The University of Calgary under the leadership of Dr Alan Hildebrand is responsible for the Near-Earth Space Surveillance (NESS) mission for asteroid science operations. DRDC is responsible for the detection and tracking of Resident Space Objects (RSOs) as part of the High Earth Orbit Surveillance System (HEOSS) [2].

This paper will discuss the stray light requirements and performance in the context of the NESS (asteroid) mission only, as it imposes more demanding requirements.

Ground-based telescopes are making constant progress in finding near-Earth asteroids. However, performing the search from space has clear advantages especially in the ability to search the ecliptic plane at closer elongations to the Sun. Ground-based opposition searches are particularly effective at discovering certain classes of NEA that cross Earth's orbit, but nearly incapable of finding NEAs orbiting completely within Earth's orbit- the Atira class. The Atira asteroids are most efficiently discovered by searches along the ecliptic plane at low solar elongations. This search space presents challenges in stray light control.

(C) Government of Canada 2012

Space Telescopes and Instrumentation 2012: Optical, Infrared, and Millimeter Wave, edited by Mark C. Clampin,

Giovanni G. Fazio, Howard A. MacEwen, Jacobus M. Oschmann, Jr., Proc. of SPIE Vol. 8442, 84424J

(C) 2012 SPIE · CCC code: 0277-786/12/\$18 - doi: 10.1117/12.925488 


\subsection{Observation strategy}

NEOSSat will follow a dawn-dusk sun synchronous orbit, at approximately $800 \mathrm{~km}$ altitude with a period of about 100 minutes. While the satellite is orbiting the east side of the Earth it will scan east and while it is orbiting the west side it will scan west in order to avoid looking over the bright Earth limb. After a half orbit the satellite will have to perform a large and rapid slew from "east to west" (and vice versa) over the poles (Figure 2). During each half orbit, the satellite will image 6 or 8 contiguous fields (describing a $2 \times 3$ or $2 \times 4$ 'race track' pattern) twice, each with a 100 sec exposure time. Revisiting the same fields during the subsequent half orbit will allow asteroids to be identified by parallax using Earth's diameter as a baseline. The parallax method will allow quick determination of distance of the NEAs and a direct way of discriminating them from the abundant main belt population.

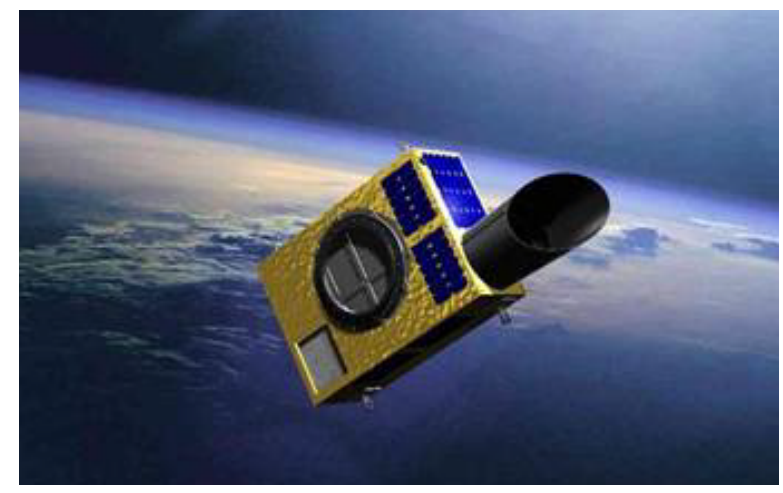

Figure 1. Depiction of NEOSSat in orbit.

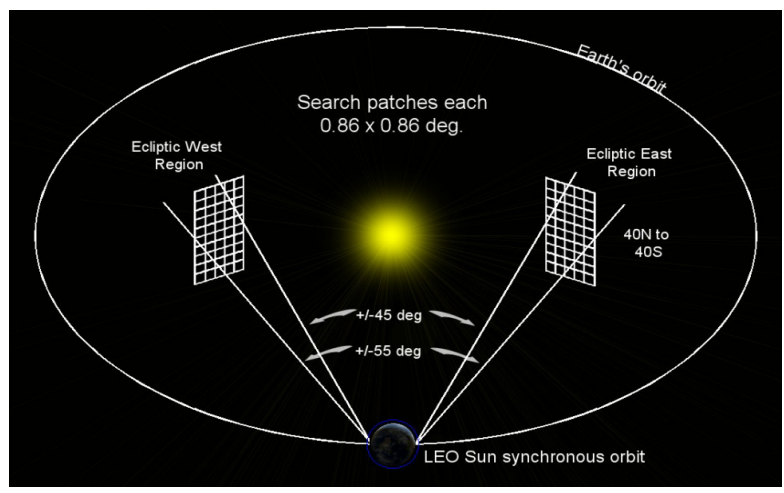

Figure 2: A representation of the search strategy for NESS (not to scale).

Two of the limiting factors in search completeness are how well stars (stellar clutter) may be removed and how well the image background can be determined. However, regardless of how effectively these can be done, a signal threshold will exist near the noise floor below which real objects may not be reliably separated from the sky background produced by the zodiacal light with contributions from detector noise and stray light.

The discovery productivity of NESS is highly sensitive to the telescope's and imager's limiting magnitude. The NESS limiting magnitude performance was simulated using several sets of assumptions and excludes scattered light. Limiting magnitudes will range from approximately 19.5 to 20.5 across the survey regions with 100 second exposures, a PSF FWHM of 1.75 arcseconds, a gain setting of 1, and assuming sub-pixel pointing stability and no object trailing losses.

Results in Figure 3 indicates a dramatic rise in expected discoveries as the limiting magnitude is lowered, with each halfmagnitude increase in limiting magnitude results in approximately $50 \%$ more asteroids than the previous half magnitude interval. This increase partly reflects the asteroid size distribution function, but also reflects the fertility of the near-Sun look angle. Aside from telescope characteristics the zodiacal light is an inescapable background in visible light in this region of the sky. This analysis demonstrated the importance of keeping noise sources (such as dark current) and stray light to a minimum on overall mission performance. 


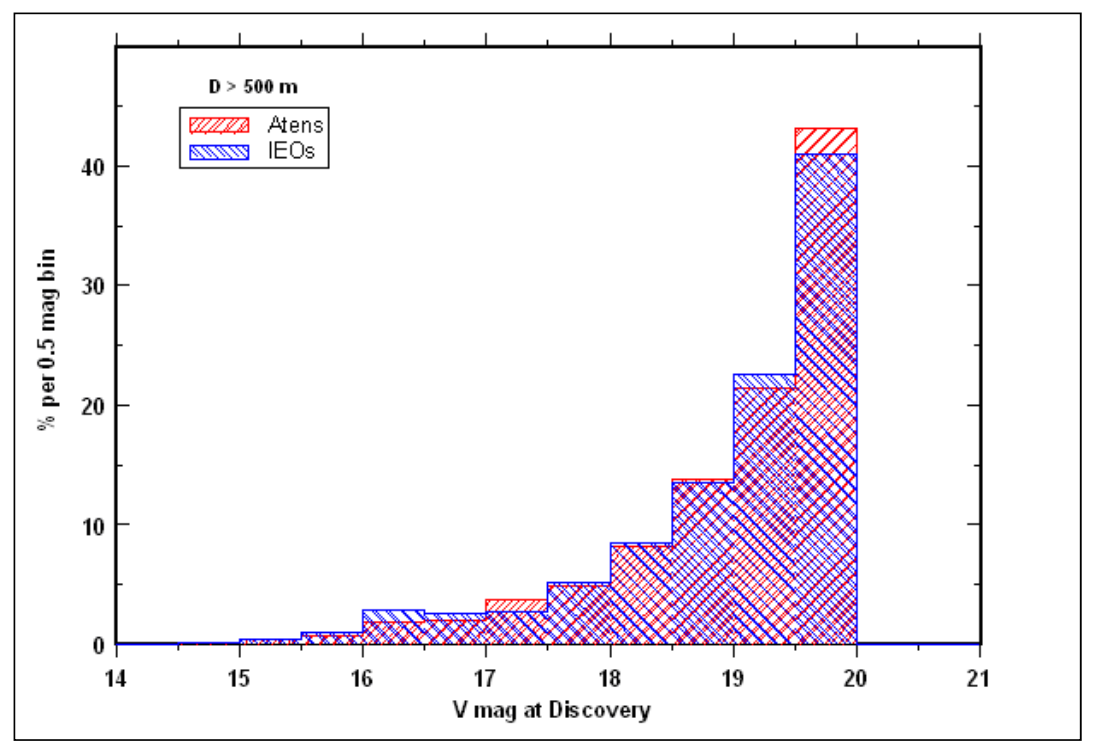

Figure 3. Sensitivity of limiting magnitude of detection. V magnitudes for Aten and IEO (Atira) class discoveries for D $>500 \mathrm{~m}$. Each half-magnitude bin contains $\sim 50 \%$ more asteroids of this size than all the brighter bins in the $\sim 19$ to 20 mag range.

\section{Spacecraft operational scenarios}

During the development Phase $\mathrm{C}$ of the mission, ten specific telescope pointing scenarios were defined and used as test points to evaluate stray light performance. For each scenario the telescope pointing was defined by Solar Elongation (Yaw), Solar Ecliptic Elevation (Pitch) and Roll Angle. In our analyses the roll angle becomes a variable. A specific scenario described below was used to perform two independent analyses. It places the satellite in a challenging position, with exposure to Earth shine at the summer solstice over the northern hemisphere. The baseline configuration used for the analyses and results presented in this paper is illustrated in Figure 18. The coordinates for this scenario are: solar elongation $45 \mathrm{deg}$; ecliptic pitch: $20 \mathrm{deg}$; roll angle nominal: $-19 \mathrm{deg}$.

\section{NEOSSat TELESCOPE}

\subsection{Optical specifications}

The optical telescope is an F/6, $15 \mathrm{~cm}$ aperture Rumak-Maksutov (Figure 4), a direct heritage from MOST [1]. However, it is optimized to provide a sharper focus and a tight PSF. Furthermore, the vignetting and distortion on the imaging CCD are designed to be minimal. The effective PSF is also a function on spacecraft pointing stability during exposure; the requirement is 0.5 arcsecond 1-sigma over $100 \mathrm{sec}$ (the pointing stability has already been demonstrated on MOST mission[3]). The F/6 system provides a square field of view of $0.85 \times 0.85$ degrees on each CCD. The total maximum field angle is 1.2 degrees. 


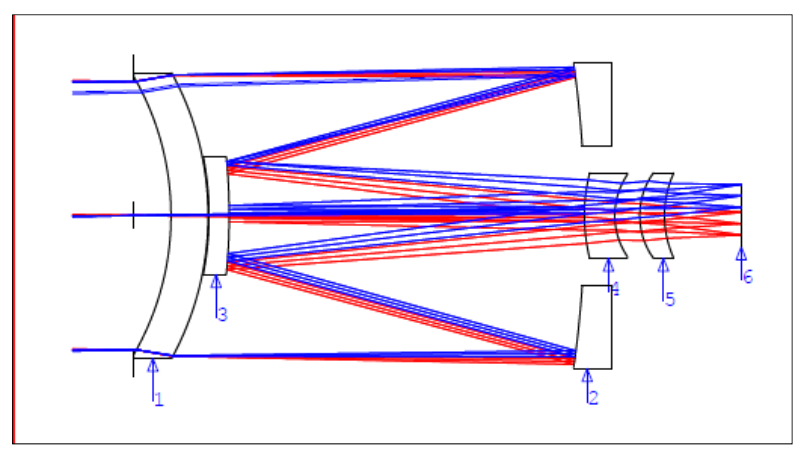

Figure 4. NEOSSat optical design Maksutov with 2 field lenses.

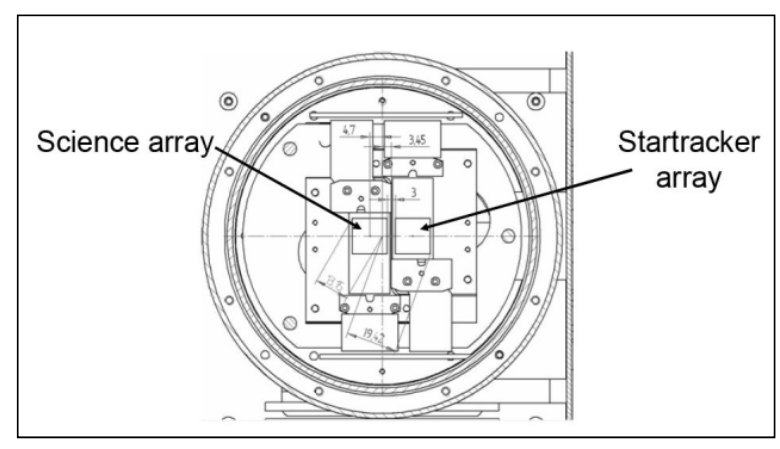

Figure 5. Focal plane with two butted CCDs.

The focal plane is shared by two CCDs (Figure 5). One CCD is used as the star tracker and operates at relatively fast update rates (about $1 \mathrm{~Hz}$ ). The second CCD is used for the science and typically calls for 100 sec exposures. Using the same telescope for the star tracker and the science imager has obvious advantages in boresighting precision and reduced system complexity.

The optical prescription was developed during Phase A of the project; field lenses were added for field flattening and reduction of distortion. Distortion (image mapping) is reduced to less than 1 pixel in order to minimize image processing of the star tracker. A tolerance analysis was conducted in Phase $\mathrm{C}$ to support opto-mechanical design and manufacturing of the optical and opto-mechanical components.

Table 1. Telescope requirements and specifications.

\begin{tabular}{|l|l|}
\hline Telescope & $15 \mathrm{~cm}$ diameter, F/6 Rumak-Maksutov \\
\hline Science FOV & $0.85 \times 0.85 \mathrm{deg}$ \\
\hline Limiting magnitude & 19.5 to $20.5 \mathrm{v}(100 \mathrm{sec}$ exposure $)$ \\
\hline PSF & $<1.5$ pixel \\
\hline Bandwidth & CCD back-illuminated with a mid-band anti-reflection coating, 300 to $1100 \mathrm{~nm}$ \\
\hline Imager & CCD back illuminated AIMO device E2V 47-20,1024x1024 (13 um) pixels \\
\hline Pixel scale & 3 arcsec/pixel \\
\hline Pointing knowledge & 1.2 arcmin $(2$ sigma) \\
\hline Stability & 0.5 arcsec for 100 second exposure \\
\hline Cadence & 288 images per day \\
\hline
\end{tabular}

A shutter is introduced between the two field lenses. The main purpose of the shutter is to protect the imagers from damage from direct solar exposure in the event of loss of attitude control (fail safe mode). The telescope does not have a door. The shutter will also serve for dark image calibration.

The opto-mechanical design is derived from that of the MOST payload. The telescope is athermalized and does not have a focus mechanism.

\subsection{Straylight requirements}

From space it is possible to observe at very low solar elongation given appropriate solar shielding at the entrance of the telescope. Being in low Earth orbit and needing to search near the ecliptic requires pointing the telescope at low elevation from the Earth's limb or surface. There are situations when light from the bright Earth can scatter into the telescope. This necessitated the design of a long external baffle (Fore Baffle) described in the next section. Simulations of signal and noise performed in Phase A set a requirement for mean stray light to be less than $10 \%$ of the zodiacal background signal level on the detectors as follows: "The scattered light from Earthshine shall be reduced to less than $10 \%$ of the sky background in the imaged portion of the sky over $90 \%$ of the orbit when the science instrument is pointed east and west of the planet in directions to at least part of the survey region of 45 to $55^{\circ}$ helioecliptic longitude 
between $40^{\circ} \mathrm{S}$ to $40^{\circ} \mathrm{N}$ ecliptic latitude." (There are also requirements for scattered light from moon and Venus, but these are more circumstantial and not analysed here.)

\subsection{External and internal baffle function}

The NEOSSat instrument requires a high performance baffle system to reject off-axis stray light for the Maksutov telescope. The fundamental design of the various baffle components was developed in the concept study and refined in Phase $\mathrm{C}$ while accommodating the practicalities of fabrication. The independent stray light analyses described here resulted in further refinements. The final design and specific features are illustrated in the figures below.

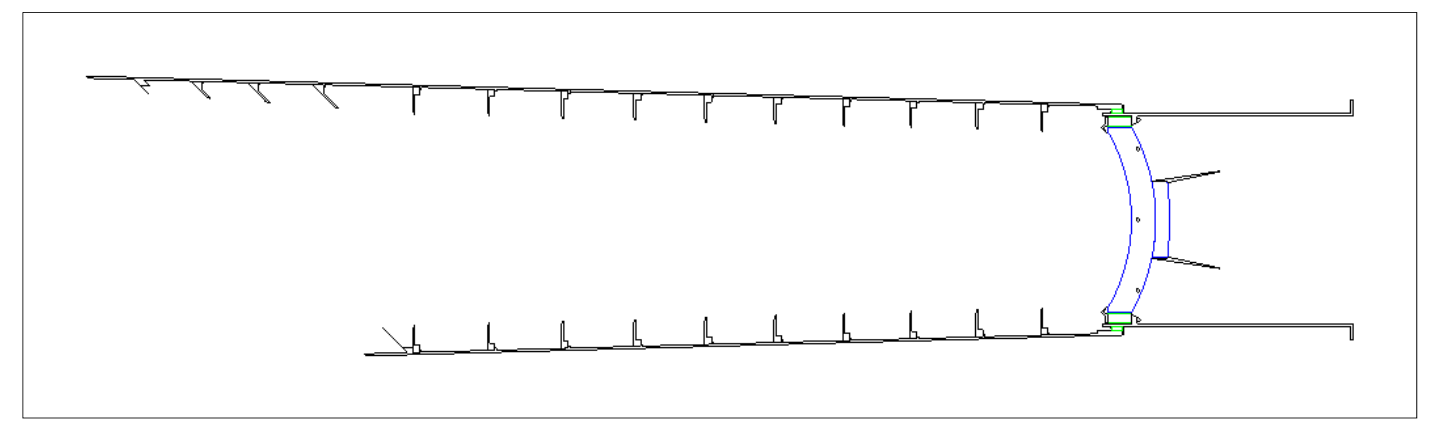

a)

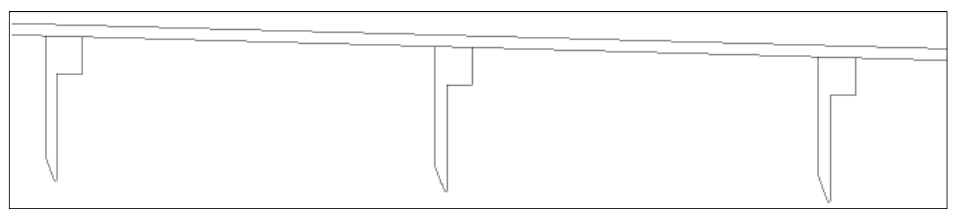

b)

Figure 6. a) Internal structure of the external baffle (Sun Shield and Fore Baffle). Corrector lens of the Maksutov appears at the right. b) Details of the vane geometry.

The stray light analysis must consider any component that has a surface that may be illuminated by imaging or stray light signals. All such components have been included in the models. The most prominent of these is the single stage fore baffle, which includes an integrated beveled Sun Shield and thirteen internal vanes. The fore baffle is situated at the front of the telescope where it attenuates stray light from off-axis light sources such as the Sun and the Earth. The telescope itself contains numerous additional features designed to help attenuate stray light (primary and secondary mirror baffles, telescope tube and apertures).

Table 2. First order parameters for the conical baffle portion of the Fore Baffle (excludes the Sun Shield).

\begin{tabular}{|l|l|}
\hline Length of conical baffle (excluding Sun Shield) $(\mathrm{mm})$ & 630.431 \\
\hline Distance from baffle exit to Corrector vertex (mm) & 22.859 \\
\hline Baffle rejection angle (degrees) & 15.5 \\
\hline Half FOV (degrees) & 1.175 \\
\hline FOV margin (degrees) & 0.4 \\
\hline HFOV with margin (degrees) & 1.575 \\
\hline Vane aperture at baffle entrance (mm) (Vane 10) & 95.779 \\
\hline Baffle skin cone angle (degrees) & 1.575 \\
\hline Exit Aperture diameter (Pupil) / margin (epsilon) (mm) & $157 / 14$ \\
\hline Number of Vanes & $10(+3$ with Sun Shield) \\
\hline Baffle Wall Thickness (mm) & 2 \\
\hline Coating & Aeroglaze Z306 \\
\hline
\end{tabular}


An important feature of the Fore Baffle is the Sun Shield. This is formed by a bevel cut at the entrance aperture of the baffle. Its purpose is to shield the internal surfaces of the baffle from direct solar and/or Earth illumination. The 45 degree bevel on the Sun Shield imparts an asymmetry to the baffle. This design approach requires that the roll angle of the spacecraft be actively maintained so as to present the Sun Shield at the optimum angle with respect to the Sun and the Earth.

Most internal surfaces in the instrument are black. Smooth surfaces are painted with Lord's Aeroglaze Z306 paint (http://www.lord.com) while surfaces with fine detail like the fine treaded interior of the secondary mirror baffle are black anodized. (The paint would otherwise fill the threads and round off sharp edges).

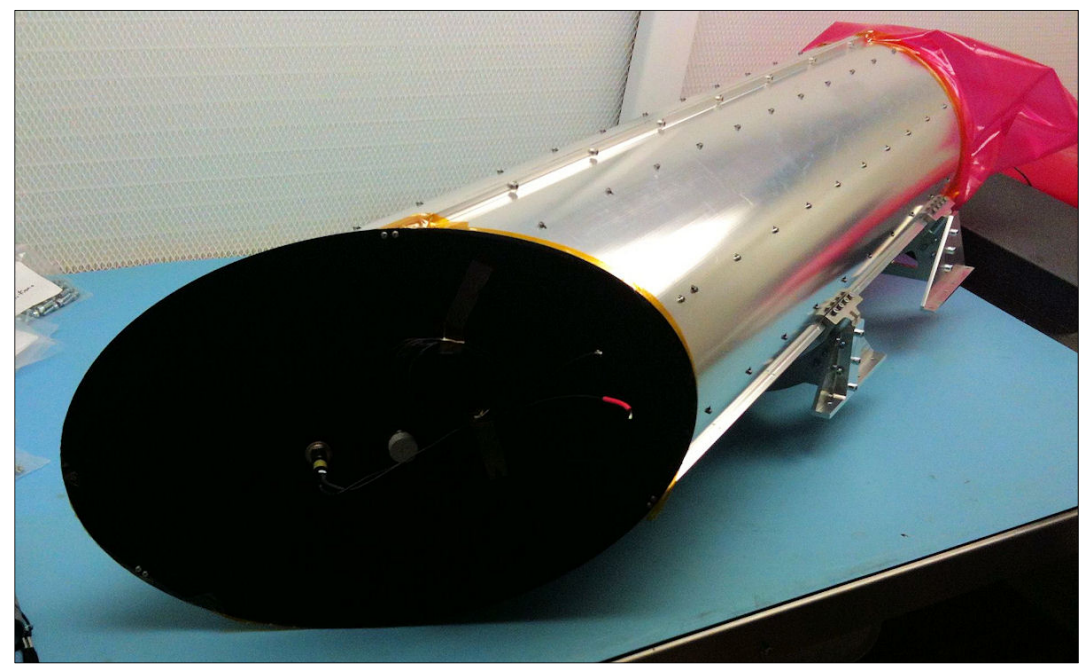

Figure 7. Photo of the assembled Fore Baffle showing a protective cover and dust bag, prior to integration to telescope and spacecraft.

\section{STRAYLIGHT MODELLING APPROACH}

The scattered light requirements dictated an attenuation of $10^{-12}$, expressed as the Normalised Detector Irradiance (see Section 4). Baffle testing was considered, but this level of attenuation is extremely difficult to measure in the lab, even with very strict environmental control in a dark clean room. Testing also adds risk due to handling of the flight unit. It was decided that performance of the baffle would be determined by analysis only.

Stray light modelling is a challenge for a complex optical system, particularly one with very high attenuation. Results are subject to statistical error and are sensitive to errors in the model. Assumptions and limitations inherent in any specific software analysis packages could also play a role in the final results. It was decided that performing two independent analyses using two different software packages would provide a higher level of confidence of the results. To improve accuracy, all opto-mechanical geometries were imported from mechanical CAD designs into the optical analysis software. Surface properties were assigned using measured scatter data.

The two stray light analyses were conducted in parallel by two independent optical engineers, each using a different software package. A detailed project plan was agreed on before any analysis work was started. A high-level breakdown of the project plan follows:

1. Verification of model geometry

a. Import CAD geometry / Generate additional required geometries

b. Verify that the models are geometrically identical in both software environments

2. Verify scatter model characteristics across software packages

a. Build and verify a "virtual scatterometer" 
b. Compare ray aiming performances over LightTools and Zemax

c. Compare measured BSDF scatter data

3. PST Comparison of subassemblies

a. PST data comparison for Fore Baffle

b. PST data comparison for Telescope

c. PST data comparison for full system

4. Full system simulations

a. Apply surface scatter properties to all surfaces

b. Set up Solar geometry and simulations

c. Run Solar illumination simulations

d. Set up Earth geometry model and simulations; run simulations

e. Run Earthshine stray light simulations: Forward vs. Reverse simulation methods

5. Review and compare analyses

The project structure forced the parallel analysis to progress in a lock-step manner. Numerous milestones were established, at which time the results of the analyses were compared and reviewed. The successful completion of each milestone was followed by a review of the next steps as prescribed by the original analysis plan. In some cases these steps might be modified based on results and with the agreement of all parties. Significant differences triggered additional investigations to discover their source and reconcile the results.

When a disagreement occurred, the project plan dictated that it be resolved before moving onto subsequent steps. In such cases the two analysts would review their methods in order to discover the source of the discrepancy. More challenging problems were solved by simplifying the particular analysis and conducting more fundamental tests until the source of the discrepancy was discovered. Regardless of the source of the error, they were caught primarily because of the parallel and independent structure of the analysis program.

The two analysts brought different approaches to many of the calculations performed throughout the process, and these differences in approach were not discouraged. Modeling approaches were discussed prior to each step. The two software packages each brought somewhat different tool sets and algorithm implementations. This frequently required different modeling strategies to calculate a particular result. Diverse modeling approaches and software factors contributed to an increased level of confidence in the performance predictions for the NEOSSat stray light performance.

\subsection{Software tools}

The two software packages used for the stray light analysis were LightTools Version 7.2 published by Optical Research Associates, now a part of Synopsys (http://www.opticalres.com) and Zemax Version July 11, 2011, published by Radiant Zemax LLC (http://www.radiantzemax.com). Both of the software packages are mature products that share a number of very similar capabilities. As mentioned previously, there are also a number of important differences between them. Some of these differences impacted the modeling methods applied in the course of the stray light analysis.

\subsection{Model geometry verification}

The parallel stray light analysis strategy relies on the assumption that the two model geometries be identical. To satisfy this requirement identical model geometries were imported into both software packages. The geometries represented the complete NEOSSat opto-mechanical assembly and were the same representations delivered to the mechanical fabricators. The geometries were supplied by the instrument's mechanical design team (Spectral Applied Research, Toronto Ontario, Canada). The data was in STEP format, which can be imported into both stray light packages. The optical elements were entered directly from the design prescription.

In spite of the common source for the imported model geometry, it was decided that verification was still required to ensure that they were imported and interpreted identically in each package. Unfortunately, direct dimensional comparison of the two models was not practical as STEP model representations do not lend themselves to easy dimensional comparison. The two software packages, while providing true 3D geometry environments, do not provide the rich sets of measurement tools that might be found in a good mechanical CAD package. In order to overcome this challenge a strategy was devised to compare geometries by tracing single rays and noting the intercept locations as the 
ray propagated through the system model. All surfaces (save for the refractive optical components) were assigned with an ideal specular reflectance property. A number of single rays were traced, targeting critical features such as radiused vane edges and threaded surfaces. Ray launch coordinates and orientations were shared between the analysts and the ray segment coordinates tabulated in spreadsheets with the help of built in data tabulation capabilities in the stray light packages. This approach made it rather easy to compare the geometries of all of the parts that were intercepted by each traced ray. An image that illustrates the method is presented in Figure 8. All regions of the models were probed by rays in this manner. The excellent correlation between the results in both the Zemax and LightTools models resulted in a high level of confidence in the similarity of the model geometries.

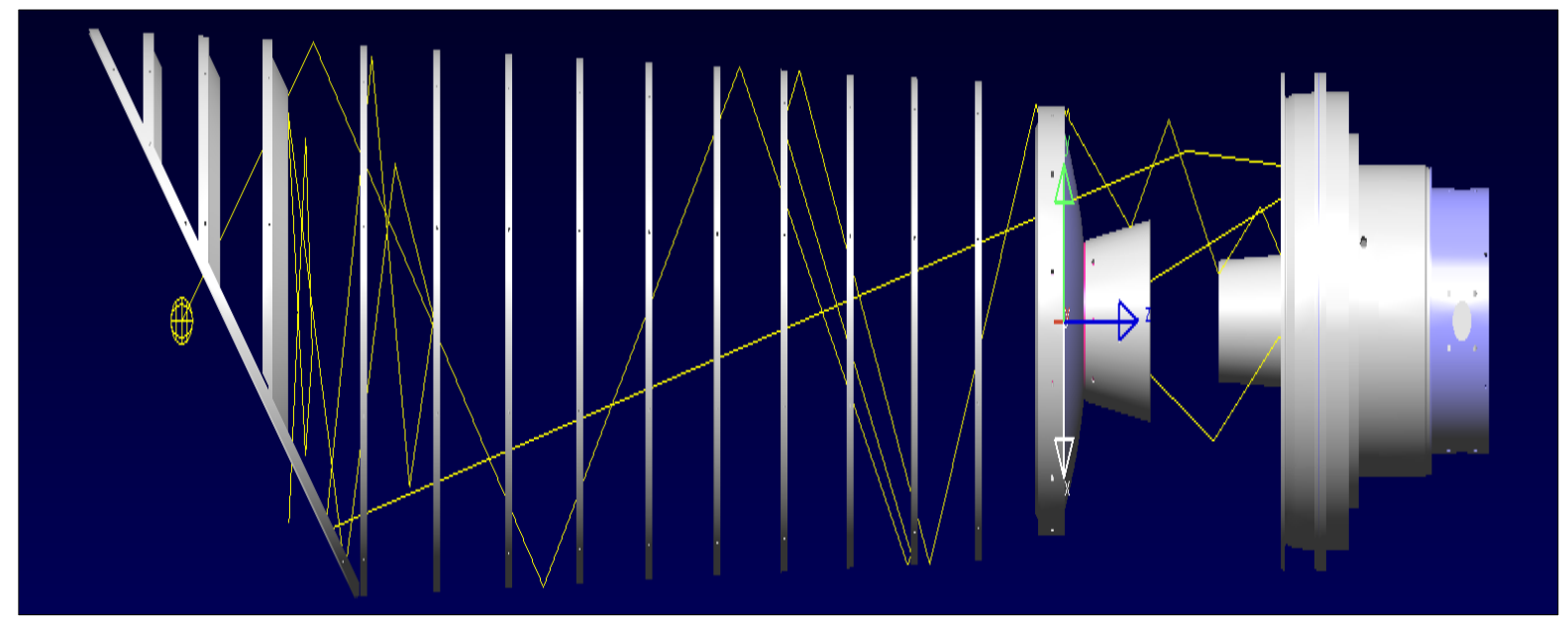

Figure 8. Specular ray trace path correlation. In order to verify the geometry of the two models, all mechanical surfaces were assigned a specular reflection property. Single rays were traced from known locations and angles and the reflection intercepts tabulated. These tabulations could then be compared. The figure shows a single ray launched from the mouth of the Fore Baffle. Note that the walls of the Fore Baffle and the telescope are not visible in these images, although they are active as can be seen from the ray paths.

\subsection{Surface properties and assumptions}

Throughout all phases of the project considerable attention was paid to assigning appropriate scattering properties to each surface, and that the two software packages interpreted those properties in a similar manner.

In the model verification stages, which included preliminary full system PST studies, the primary objective was to verify the similarity of the models across the two software environments. Only simple "native" scatter models were applied during these activities. Such properties are analytic and are implemented natively by both software packages. Examples of these native models include Lambertian scatter models with a fixed reflectance, specular reflection, and pure absorbing surfaces.

After the verification stages were complete, more accurate surface scatter models were assigned. Many of the mechanical surfaces in the NEOSSat instrument were specified to be coated with a black anodize or with Z306 paint. These scatter properties were measured from prepared samples and represented in software through Bidirectional Scatter Distribution Function (BSDF) definitions. A small number of surfaces (i.e. gold coatings), were assigned more general analytic scattering properties provided by the software packages. In order to ensure uniformity of the two models, scatter property assignments were tabulated and shared through spreadsheets.

The Z306 and black anodize coating scatter properties were measured by Radiant Imaging (RI) under contract. (Radiant Zemax: http://www.radiantzemax.com/) The Z306 sample was taken from the same lot of paint purchased for application on the NEOSSat instrument. Two anodized aluminum samples were fabricated by the supplier contracted to provide parts for the instrument.

The measured data was delivered in RI's proprietary "IS" data format. The measured scatter data was read into RI's ISSA (Imaging Sphere for Scatter and Appearance measurement) software package. The IS-SA software was used to 
export the data into (BSDF) data sets in formats compatible with LightTools and with Zemax. (This required two different formats.) The resulting BSDF files were then imported into Zemax and LightTools and assigned to the appropriate surfaces.

\subsection{Scatter models verification}

As with all other aspects of the parallel stray light analysis, the scatter models underwent a verification process in order to confirm that both software packages interpreted and simulated the measured data in the same way. Leveraging the modeling capabilities of the two software packages, a simple "virtual scatterometer" was set up in each environment. A simple Lambertian scatter property was used to confirm the operation of the two models, after which the measured data was analysed and compared. The correlation between the results was very good.

The virtual scatterometer exercise provided an opportunity to compare the implementations of importance sampling (sometimes called ray aiming) by the two packages. Importance sampling can significantly improve the efficiency of Monte Carlo ray trace simulations by only tracing scattered rays into a predetermined solid angle or at a specified surface. This approach ignores rays that might be scattered in directions that the analyst knows will have no contribution to the stray light calculation at hand, thereby making the calculation significantly more efficient and accurate, and saving a great deal of computation time.

The implementation of importance sampling differed between the two packages and the virtual scatterometer strategy provided and reliable method to work out strategies to compensate for these differences and obtain accurate, wellcorrelated scatter results.

\section{Coordinate system}

Independent analyses required careful attention to coordinate systems. A spherical coordinate system was adopted for the stray light analysis (Figure 9). The orientation of the reference coordinate system with respect to the NEOSSat instrument is shown in Figure 15. Note that the elevation angle $\theta$ (theta) made with respect to the optical axis, and azimuth angle $\phi$ (phi) rotates about the optical axis. The direction cosines $1, \mathrm{~m}$, and $\mathrm{n}$, are the projection of the ray, drawn to a unit sphere, onto the $\mathrm{x}, \mathrm{y}$, and $\mathrm{z}$ axis, respectively. They are given by:

$$
1=\sin \theta \cos \varphi ; m=\sin \theta \sin \varphi ; n=\cos \theta \quad \text { Equation } 1
$$

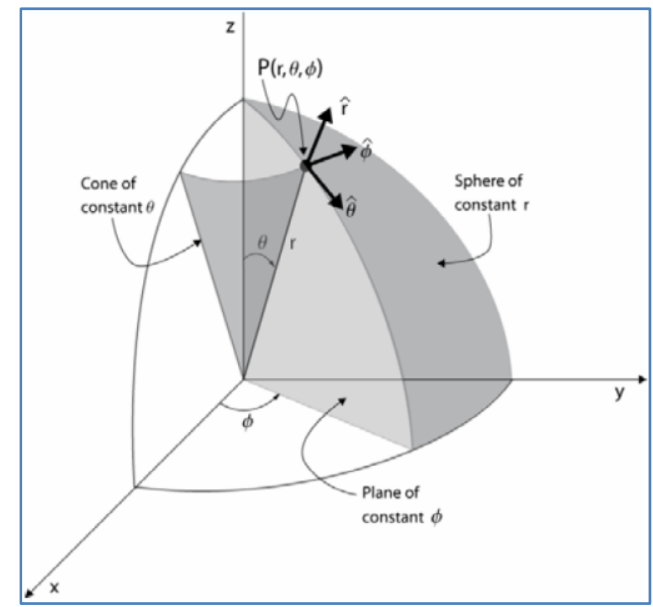

Figure 9. Spherical coordinate system. (Adapted from: http://www4.wittenberg.edu/maxwell.)

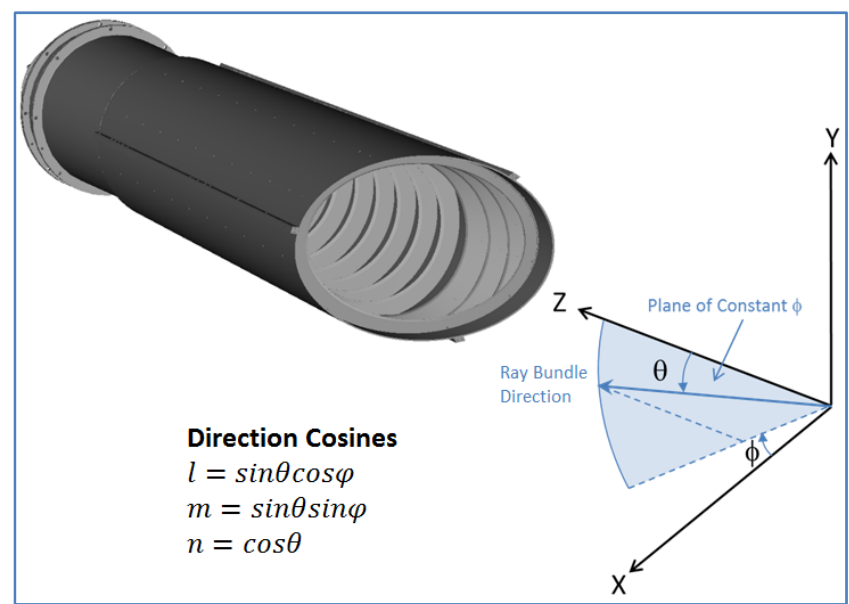

Figure 10. NEOSSat optical coordinate system showing source spherical angles. Note that the entrance aperture of the Fore Baffle is cut at a $45 \mathrm{deg}$ with respect to the optical axis. The normal to the plane of the entrance aperture has an azimuth value of zero and an elevation angle of -45 degrees. 
The term "roll" is used here to describe the instrument's azimuthal orientation during operation. Roll is the rotation of the instrument about the optical axis. It is used to orient the Sun Shield for optimum rejection of unwanted illumination from the Sun and the Earth, and is critical in achieving the stray light performance targets set out for the mission.

For accurate modeling of the operational requirements of the instrument, the optical coordinate system described here was related to the satellite and ecliptic coordinate systems. This is described in a subsequent section of the paper.

\section{RESULTS AND OPERATING CONSTRAINTS}

\subsection{PST of subassemblies and of full system}

The point source transmission (PST) is a well-accepted stray light figure of merit for optical systems. It is expressed as a ratio, and there are several variants. The normalized detector irradiance (NDI) is used here. The NDI is defined as the irradiance at the detector due to a point source divided by the irradiance at the entrance port due to the same point source. The point source is assumed to be at an infinite distance, and so is represented by a collimated ray bundle that fills the aperture.

$$
\operatorname{PST}(\varnothing, \theta)=\frac{\text { Detector Irraddiance }(\varnothing, \theta)}{\text { Port Irraddiance }(\varnothing, \theta)} \quad \text { Equation } 2
$$

In keeping with the "small steps" strategy employed throughout the project, the PST analyses were first carried out on the Fore Baffle and Telescopes independently. Only after these studies were satisfactorily completed was a full system PST analysis completed.

\section{Fore Baffle PST analysis}

The first subsystem performance testing and comparison was carried out on the Fore Baffle system. This subsystem includes the Fore Baffle, which comprises the bevelled Sun Shield, the conical Fore Baffle shell, and all of the internal vanes. Simple analytic surface scatter properties were applied for this stage of the program.

Initial PST runs were conducted through a zero azimuth slice in order to verify common source and receiver geometries and other simulation parameters. Routines were written and refined to generate results through long (i.e. multiple hours) simulation run times. Once the simulation parameters were worked out, PST data was generated for azimuthal angles of $0^{\circ}, 30^{\circ}, 60^{\circ}, 90^{\circ}$, and $180^{\circ}$, with $5^{\circ}$ off-axis resolution. The results are shown in Figure 12 and have excellent correlation. The conclusion is that the Fore Baffle models are very similar and the simulation methods and analyses are also well correlated.

The Fore Baffle PST analysis laid the groundwork for the next stages of the program. Coordinate and sign conventions were established, as were data collection and tabulation methods. An increased familiarity and appreciation of the similarities and differences between the two software packages was developed at this stage.
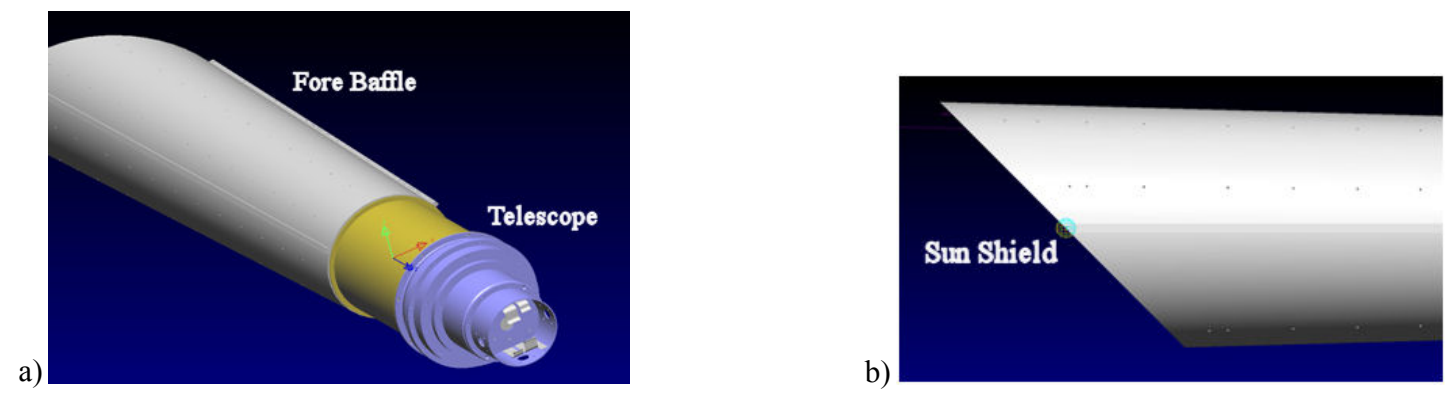

Figure 11. a) The NEOSSat instrument is analyzed in two parts: the Fore Baffle (gray) and the Telescope (yellow and blue). b) The front of the Fore Baffle is designed with an aperture cut at 45 degrees with respect to the optical axis. This region of the Fore Baffle is called the Sun Shield, and is an integral part of the Fore Baffle. 


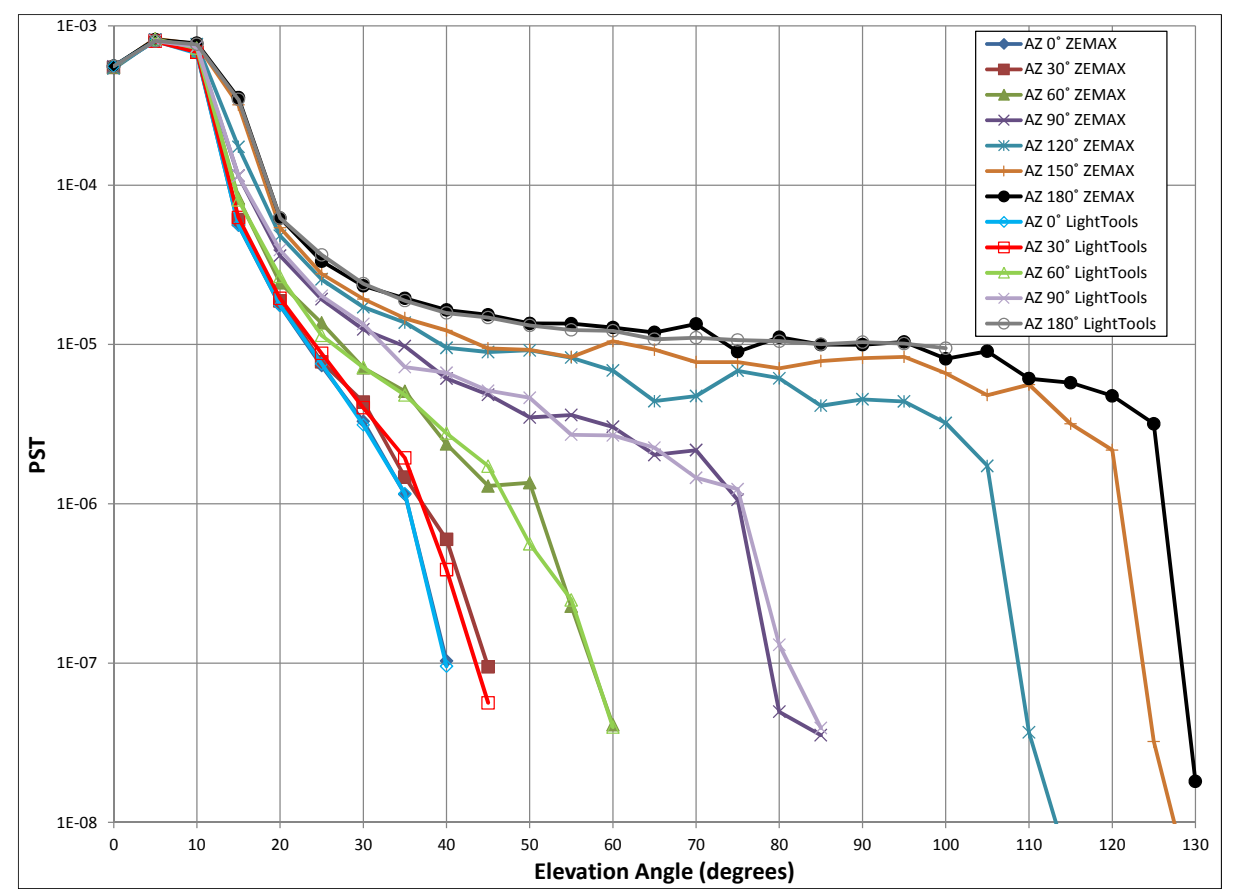

Figure 12. Fore Baffle PST curves generated in LightTools and Zemax.

\section{Telescope PST analysis}

The telescope PSTs were generated by placing the source rays just before the corrector baffle ring. No Fore Baffle shading or scatter is considered. As with the Fore Baffle analysis, native coatings were used in the telescope calculation. Black surfaces, RTV, and gaskets were assigned Lambertian 10\% reflective coatings. Smooth metallic and gold surfaces were assigned specular coatings of $50 \%$ and $80 \%$ reflectance, respectively. Mirror reflectance and lens transmittance were unity. Mirror and lens edges were $100 \%$ absorbing.

PST data was generated for azimuthal angles of $0^{\circ}, 30^{\circ}, 60^{\circ}$, and $90^{\circ}$, with $5^{\circ}$ off-axis resolution. With little exception, the LightTools and Zemax results are in good agreement as shown in Figure 13. For azimuthal angles of $0^{\circ}, 30^{\circ}, 60^{\circ}$, the LightTools PST is marginally higher for off-axis angles from $60^{\circ}$ to $80^{\circ}$. The most notable departure in the PST occurs for an azimuthal angle of $0^{\circ}$ at an elevation angle of $20^{\circ}$, where the magnitude of the spike is an order of magnitude higher in Zemax than in LightTools. Further investigation showed that this spike is a result of a glint path off of the shutter ring inner edge. This was found to be caused by an asymmetrical cut-out of an internal baffle in front of the shutter, allowing the shutter ring edge to be directly illuminated at a narrow range of angles. 

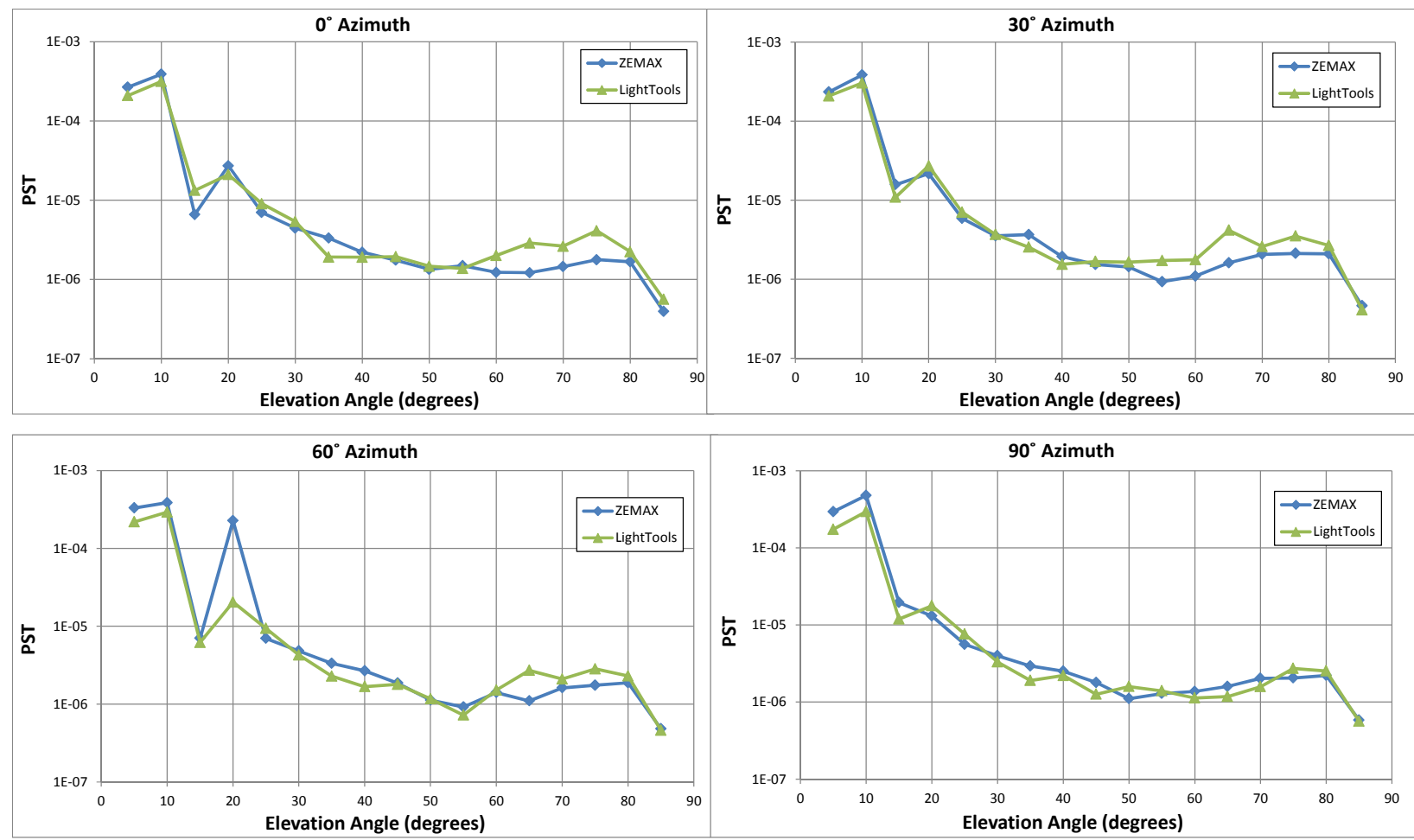

Figure 13. Telescope-only PST curves generated in LightTools and Zemax.

\section{System PST verification}

The system PST was generated in LightTools and Zemax using the same native coatings used in the telescope analysis. This time the source rays were placed in front of the Sun Shield opening thus including the Fore Baffle in the analysis. There were several differences between the two analyses in the importance sampling was applied. In LightTools ray aiming was used to control scatter light from the vane tips - the Zemax model did not use ray aiming for these tips. To obtain adequate representation of the tips in Zemax, ten rays were scattered for each incident ray. For all other Fore Baffle surfaces, only one ray was scatter per incident ray. Ray aiming was used in both programs for the primary and secondary baffle inner surfaces. Ray aiming was also used in Zemax to scatter rays from the primary mirror baffle ring. No ray aiming was used for this object in LightTools.

PST data was generated for a single azimuthal angle of $-35^{\circ}$, with elevation angles of $18^{\circ}, 28^{\circ}, 38^{\circ}$, and $48^{\circ}$. The PSTs are compared in Figure 14 by the curves identified as "baffles". Although the shape of the LightTools and Zemax PST curves are well correlated, the results are separated by constant scaling value. The difference may be explained by the different ray aiming parameters or routines used in the programs.

Also plotted in Figure 14 is the contribution due to scatter from the optics. Here, light is allowed to scatter from the Sun Shield, Fore Baffle, and Corrector Baffle Ring, then subsequently scattered from the corrector, the mirrors, and lenses to the detector. The optical contribution is about $10 \%$ of that of the baffles. Surface scatter from the optics was not included in the LightTools analysis, nor was it considered for the final system analysis presented in this paper. 


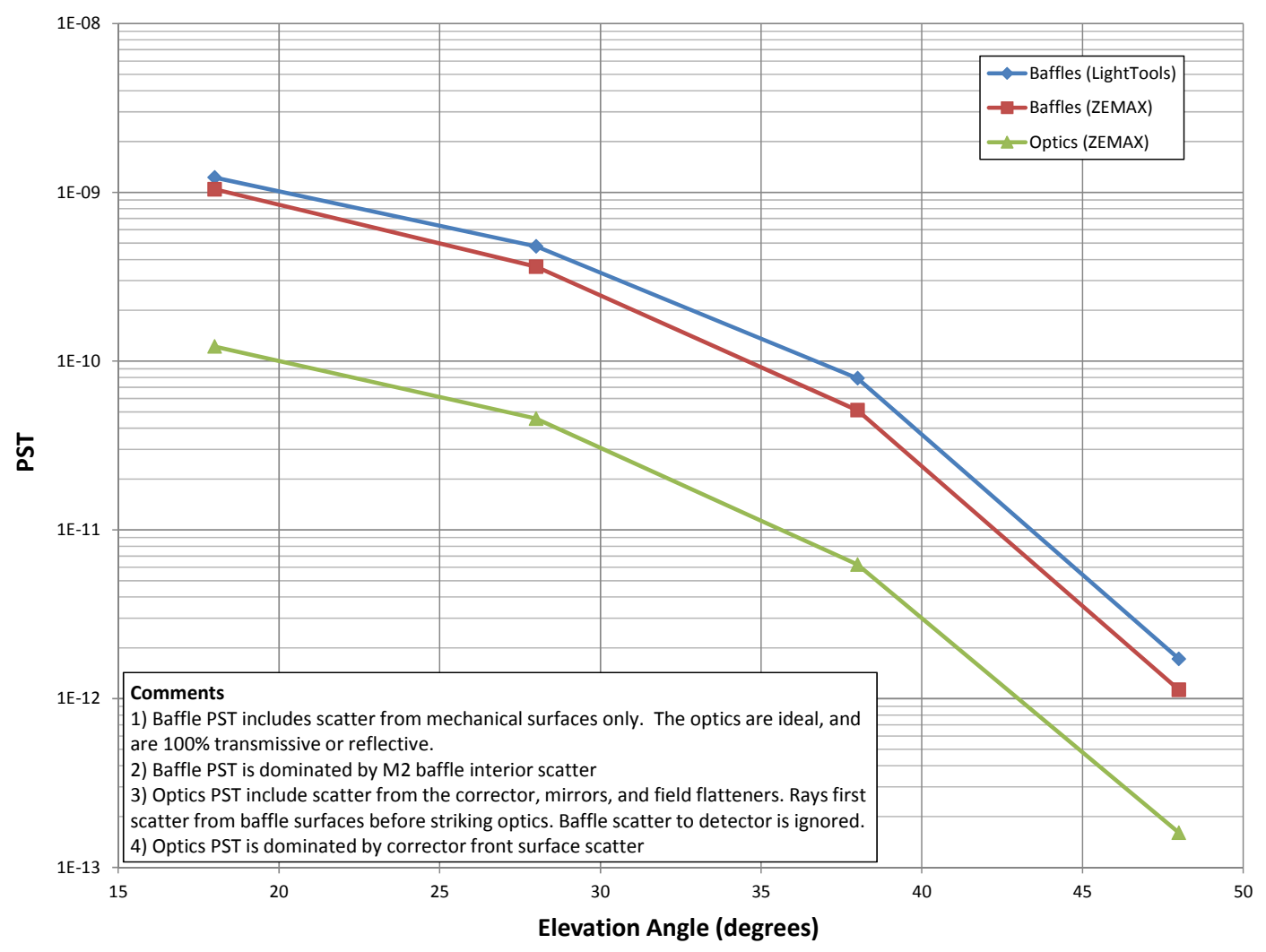

Figure 14. System PST curves generated in LightTools and Zemax.

\section{Sunshine and Earthshine analysis for a specific case}

For the solar and Earthshine analysis of the specific operating scenarios, the models were prepared by assigning the appropriate scatter properties to all of the surfaces. Most of the internal surfaces in the Fore Baffle, including the vanes, are coated with the Aeroglaze Z306 paint. A number of surfaces within the Telescope are also coated with the Z306 paint. Other surfaces are coated with a black anodize finish. All of these surfaces were assigned with the measured BSDF scatter definitions. A tabulation of all of the instrument surfaces was maintained and shared between the analysts to track surface scatter assignments.

\section{Solar and earthshine stray light analysis - mission requirement modeling}

As mentioned earlier, the stray light requirements for the NEOSSat mission were specified in the form of specific operational scenarios. The scenarios related the location of the Earth and the Sun to NEOSSat's orientation. The scenarios were specified in a multitude of parameters including ecliptic coordinates, solar elongation, satellite altitude, orbit epoch (reference date), and others. Adding to this coordinate complexity was the fact that the satellite coordinate system differed from the optical system coordinate system. To reduce the complexity and eliminate the risk of error, the prime contractor (Microsat Systems Canada Inc., Mississauga, Ontario, Canada) provided an unambiguous coordinate transformation table for each of 10 unique scenarios. This allowed the analysts to establish simple vectors in the optical coordinate system that located the Sun and the Earth. As a visual aid these vectors were included within the LightTools model. These aids were of great help in setting up and debugging simulation routines that involved translations and rotations of simulation sources. An example of this is shown in Figure 15. 


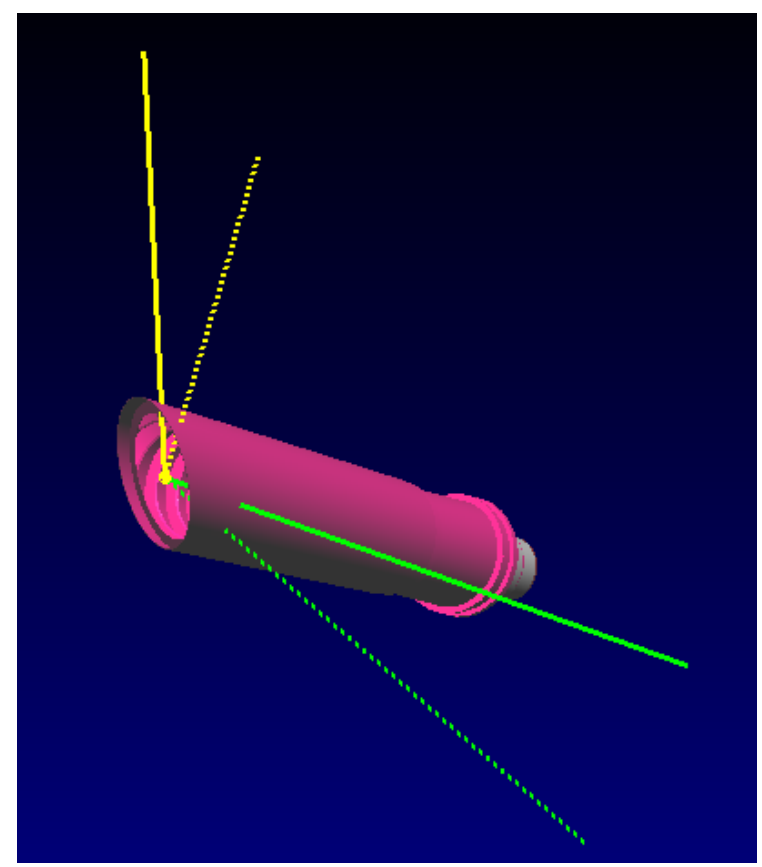

a)

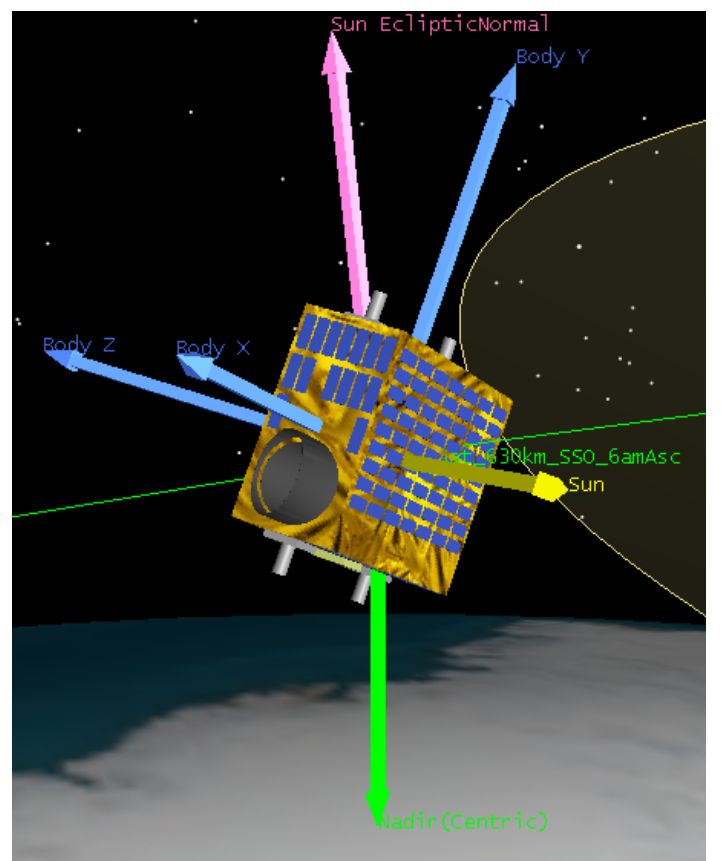

b)

Figure 15. a) Earthshine modeling in LightTools included vector indicators to show the location of the Earth (green line) and of the Sun (yellow line). The nominal vectors for each configuration (solar optimal) are indicated by dotted lines. As the model was rolled from the nominal a second pair of vectors (indicated by solid lines) was used to show the orientation of the Sun and Earth in the rolled configuration. This image shows a roll of -25 degrees from solar optimal. The addition of these vectors directly into the stray light modeling environment provided useful visual aids to verify the complex 3D geometries generated by the orbital model. b) Image of the satellite showing the vectors for Scenario 6.

\section{"Roll angle" orientation strategy}

A key part of the stray light rejection for NEOSSat is the "roll angle" of the satellite. Physical constraints limited the size of the Fore Baffle, precluding the implementation of a two stage baffle that could reject stray light from both the Sun and the Earth from any azimuth. The 45 degree angle on the entrance aperture on the Fore Baffle forms a Sun Shield, and it can be used to completely reject direct illumination of any internal surfaces by the Sun if it is oriented towards the Sun and the Sun was located at an angle greater than 45 degrees. For some configurations illumination from the Earth dominated the stray light performance of the instrument. It was found that by "rolling" the instrument about its optical axis away from its optimal sun shielding orientation so as to partially block the Earth's illumination, both the solar and Earthshine stray light performance requirements could be met. Adjusting the roll angle maintains the pointing direction of the telescope but changes the relative azimuth of the Sun and the Earth. This strategy is quite acceptable with an agile satellite such as NEOSSat.

The Stray light PST plots for the Solar and Earthshine plots show the instrument PST as a function of the roll angle. The roll angle was defined as the azimuth angle from optimum orientation to the Sun. Plots could then be used to indicate the optimum roll angle of the satellite, and the range of the acceptable roll angles.

\section{Solar illumination}

The plot for the solar-induced stray light is shown in Figure 16. The Zemax and LightTools models correlate quite well. The LightTools model shows more structure than the Zemax results. In the region between -40 degrees and -30 degrees, the values are within a factor of two of each other. This is considered to be a good degree of correlation for the two analyses. 


\section{Earthshine analysis}

The Earthshine analysis is fundamentally more complex than the solar analysis because the Earth presents a very large angular source. The Zemax and LightTools Earthshine modeling methods differed significantly. The Zemax approach integrated an array of PSTs to calculate the contribution over the angle subtended by the Earth. The LightTools model used a reverse ray trace model that traced rays from the detector to a receiver that occupied the same angular field subtended by the Earth (see for example [5]). The Zemax model performs numerical smoothing between PST calculations while the LightTools model does not.

The plot in Figure 17 presents the Earthshine results for the LightTools and Zemax models. The two plots are again very well correlated. It is interesting to point out that the Zemax and LightTools results agreed immediately after the first simulations of the Scenario 6 were complete. There were no challenges or issues in having these relatively complex but independent simulations agree with each other. This is almost certainly due to the extensive model verification process that preceded these simulations. To meet the dark sky requirement, the operating roll angle would appear to lie $<-15$ degrees.

\section{Integrated Earthshine and Sunshine analysis}

Combining the Earthshine and Sunshine stray light results together in a single plot presents a simple method for determining the roll angle "operating range" for the instrument. The operating range is the range of roll angle over which the instrument will simultaneously satisfy the solar and Earthshine requirements. Similar plots were generated for all of the other scenarios specified as part of the mission requirements.

The data are plotted in Figure 18. The plot includes both the Zemax and LightTools data. A green arrow indicator is plotted at the heart of the operating range. The range presents a region spanning approximately 10 degrees that exceeds the requirement by a factor of approximately four times.

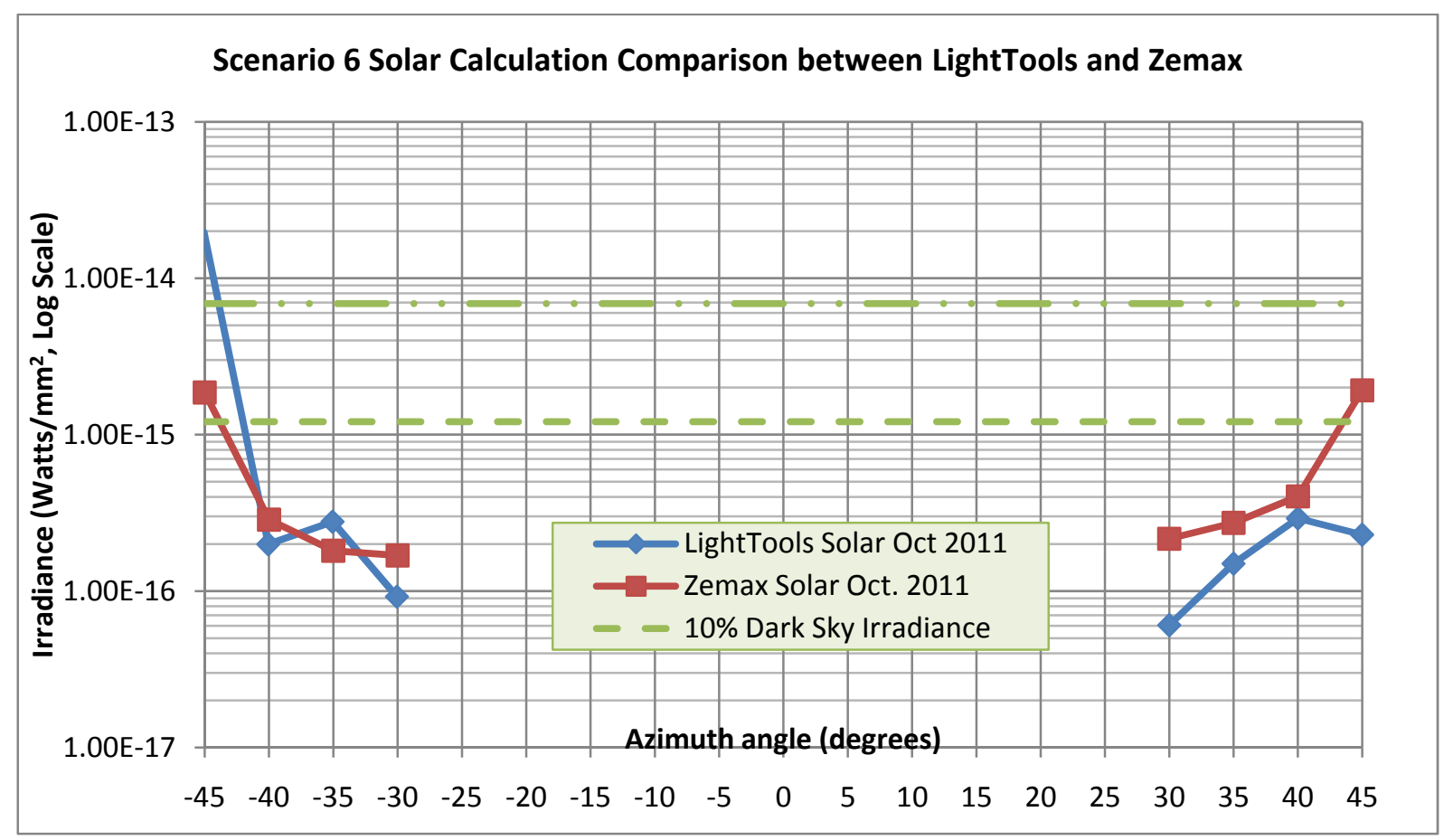

Figure 16. Comparison of results for stray light due to solar irradiance. The two analyses show reasonable correlation. Note that the gap in data between -30 degrees and +30 degrees results from the complete obscuration of the Sun from the Fore Baffle by the Sun Shield. No (geometric) rays can illuminate the internal walls or structures in this angular range. Azimuth is equivalent to roll angle. 


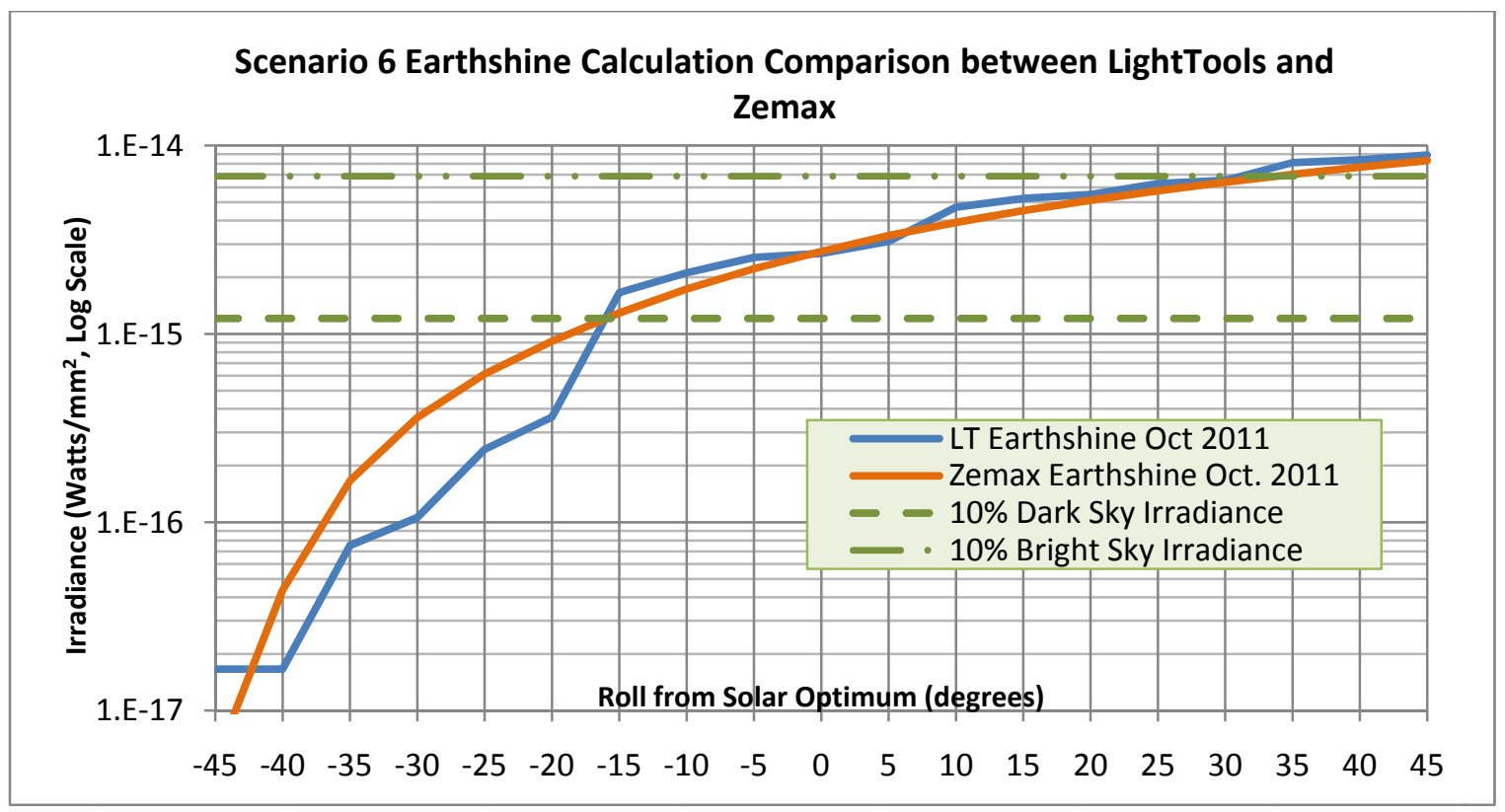

Figure 17. A comparison of the stray light results for Zemax and LightTools. The results of the two analyses show a strong correlation. The integration method results in a smoother plot. Both the LightTools and Zemax simulations were calculated at five degree angular increments.

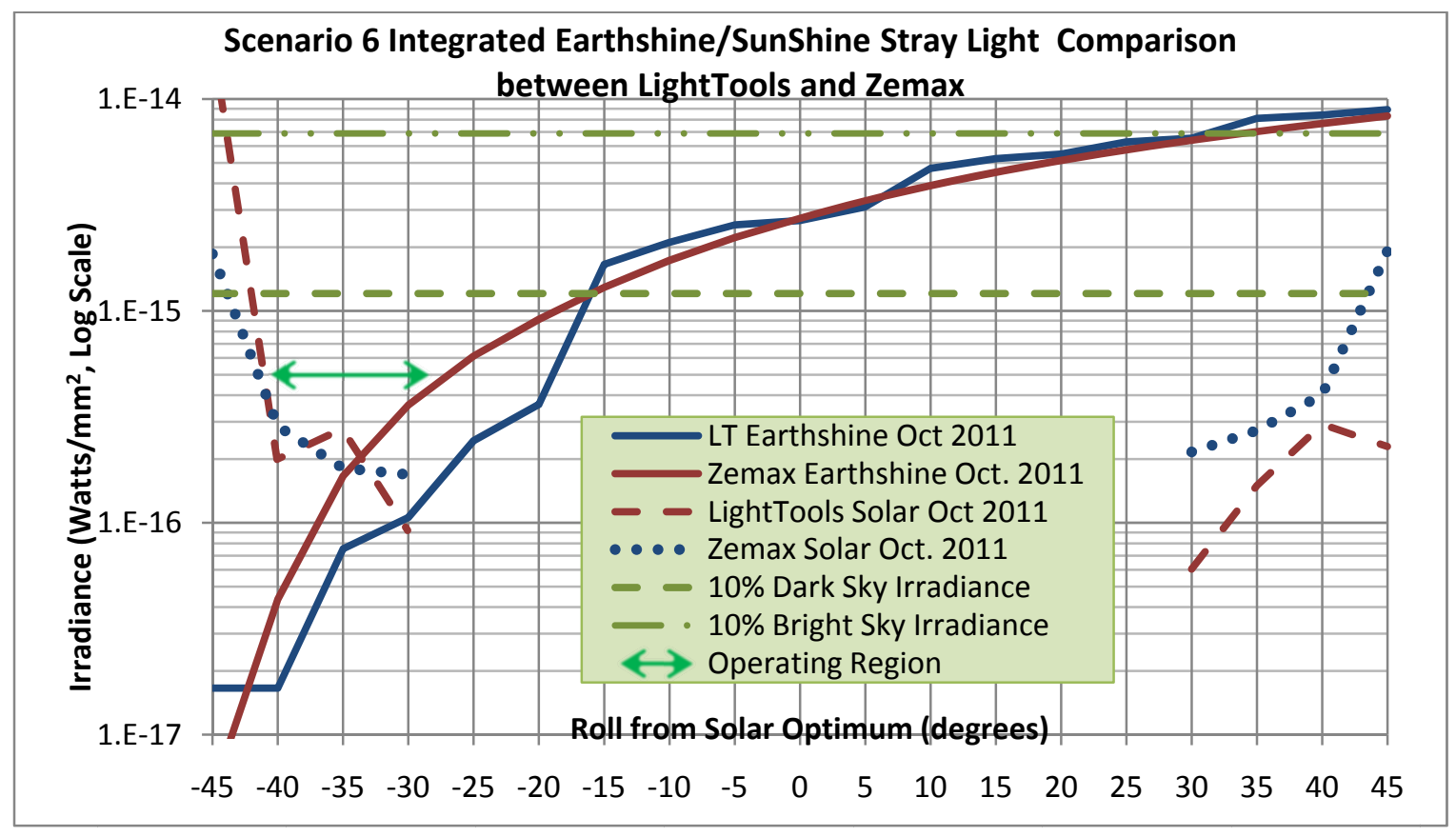

Figure 18 Results of the Scenario 6 as predicted by the two concurrent analyzes. The combined result points to an operating region (i.e. Range of acceptable roll values) that would allow the instrument to operate within the requirements. The margin at -35 degrees is approximately four times better than the dark sky threshold. The operating range extends from -40 degrees to -20 degrees 


\section{CONCLUSIONS}

The independent and parallel stray light analyses have proven to be an effective approach to verify the performance of the NEOSSat space telescope baffle. A preliminary analysis, done in the early phases of the mission, presented only a small margin towards meeting the requirements given the high stray light rejection desired. A physical test of the baffle was considered to be risky both in terms of obtaining accurate measurements of the light rejection and in the handling of the flight components. Two independent analyses were then undertaken to raise the level of confidence of the design. The analyses were conducted using different software packages (LightTools, Zemax). This approach allowed a step by step verification of the assumptions and interpretations of the software models. It is worth noting that a similar comparison between a software model with a physical test would have been more difficult if not inconclusive. The PST and stray light irradiances results presented, subject always to some statistical errors, agree within a factor of two overall, which is considered good in this type of analysis. It demonstrated with reasonable margin that the baffle has an operating range meeting the mission requirement. In the course of the work which required very detailed examination of all components by both analysts, several refinements of the design were suggested and implemented.

At the time of this writing, the payload has completed optical imaging test and is undergoing instrument signal test. The integrated spacecraft will be sent to CSA David Florida Laboratories (Ottawa, Canada) facility for TVAC and vibration testing. NEOSSat will be launched as a secondary payload on the ISRO PSLV launcher in late 2012. Stray light performance will be verified in several different scenarios during commissioning phase and compared with the analyses.

\section{ACKNOWLEDGMENTS}

The authors would like to thank several people for providing details and useful comments in the course of this work. Don Sturgeon, Spectral Applied Research, for providing the CAD models for the analyses; Charlie Hersom, Spectral Applied Research, for supporting the stray light analysis program; James Wells, Microsat Systems Canada Inc, for providing the scenarios with the coordinate transformations during Phase B, which played a key role in defining the stray light analysis modeling and the 'roll angle' strategy.

\section{REFERENCES}

[1] Grocott, S. C. O., Zee, R. E., Wells, G. J., Wessels, R., Beattie, A., Park, S., "Expanding the Capabilities of the MOST Spacecraft", ASTRO 2006 - 13th CASI Canadian Astronautics Conference, Montreal, Canada, April, (2006).

[2] Hildebrand, A.R., Carroll, K.A., Tedesco, E.F., Faber, D.R., Cardinal, R.D., Matthews, J.M., Kuschnig, R., Walker, G.A.H., Gladman, B., Pazder, J., Brown, P.G., Worden, S.P., Burrell, D.A., Chodas, P.W., Larson, S.M., Wallace, B.J., Muinonen, K., Cheng, A., "Advantages of Searching for Asteroids from Low Earth Orbit: The NEOSSat mission", Earth Moon and Planets, Vol. 95, p. 33-40 (2004).

[3] Grocott, S. C. O, Carroll, K. A., "Arc-Second Attitude Control for the NESS Asteroid/Satellite Tracking Microsat", Sixteenth AIAA/USU Conference on Small Satellites, (2002).

[4] Laurin, D., Hildebrand, A., Cardinal, R., Harvey, W., Tafazoli, S., "NEOSSat - A Canadian small space telescope for near Earth asteroid detection", SPIE Space Telescopes and Instrumentation 2008: Optical, Infrared, and Millimeter, Proc. of SPIE Vol. 7010, (2008).

[5] Pfisterer, R. N., "Clever tricks in optical engineering", Novel Optical System Design and Optimization VII, Proc. of SPIE Vol 5524, (2004). 